\title{
Insilico identification of potential antivirals and molecular dynamics against SARS-CoV2 main protease and RBD of spike protein
}

\author{
Vijayakumar Rajendran"\$, Saravanan Kandasamy", Ankita Gupta\#, Jagannathan \\ Selvaraj $^{\epsilon}$ and Shivanandappa Kukkaler Channappa ${ }^{\star}$
}

*Molecular Biophysics Unit, Indian Institute of Science, Bangalore 560 012, India

${ }^{\$}$ Department of Biochemistry, North-Eastern Hill University, Shillong 793 022, India

\#Department of Zoology, Gauhati University, Gauhati 781014, India

${ }^{\epsilon}$ Tissue culture anti-rabies vaccine section, Pasteur Institute of India, Coonoor 643 102, India

Pertussis Vaccine Section, Pasteur Institute of India, Coonoor 643 102, India

VR, SK and AG contributed equally to the manuscript 


\begin{abstract}
A coronavirus identified as 2019 novel coronavirus (COVID-19) is the etiological agent responsible for the 2019-2020 viral pneumonia outbreak that commenced in Wuhan has been declared as a pandemic by the World Health Organization. The virus is predominantly spread from person-to-person mainly through airborne, fomite, contact, and droplet from the infected patients. Also, the lack of definitive treatment is another concern that needs consideration. The novel 2019 SARS-CoV-2 enters the host cell by binding of the viral surface spike glycoprotein (S-protein) to angiotensin-converting enzyme 2 (ACE2). $\mathrm{M}^{\text {pro }}$ is a key coronavirus enzyme, which plays a pivotal role in mediating viral replication and transcription, making it an attractive drug target for this virus. Considering the importance of these two proteins in the viral infection, these were preferred as a potential drug target against Covid19. In this study, we screened potential antiviral drugs from the Pubchem database and natural antiviral agent quercetin for induced fit docking against these two key proteins. The identified top hit was further evaluated through molecular dynamic simulations. Our results suggest that the antiviral drugs Indinavir and Famciclovir could be a potential drug against Covid19.
\end{abstract}

\title{
Keywords:
}

Covid19; virtual screening; induced-fit docking; molecular dynamics; quercetin; indinavir; famciclovir 


\section{Introduction}

The Severe Acute Respiratory Syndrome Coronavirus 2 (SARS-CoV-2) is a novel virus strain that causes a significant outbreak of coronavirus disease (COVID-19), a respiratory illness (World Health Organization 2020). SARS-CoV-2 reported first in Wuhan, China, in late December 2019 and then spread from country to country through a new travel route and declared as a global pandemic by the World Health Organization (World Health Organization 2020; Yang et al. 2020). WHO recommended frequent hand washing and maintenance of social distance to control COVID-19 disease. However, these measures are not able to prevent infection by an infected person who carries viral content. India first reported the COVID-19 case in Kerala on $30^{\text {th }}$ January 2020 and currently $14,043,176$ patients affected globally and in which $1,077,618$ patients from India alone (on $20^{\text {th }}$ July 2020) (Lathika Rajendrakumar et al. 2020).

Coronavirus come under the family of enveloped, positive-stranded RNA viruses, infecting only vertebrates or even life-threatening diseases in human. The international committee on taxonomy of the virus (ICTV) is responsible for the classification and naming of the virus (Cui, Li \& Shi 2019). Coronaviridae study group (CSG) of ICTV developed the family, classification, and taxonomy of coronaviruses. SARS-CoV-2 come under family coronaviridae family, genus beta coronavirus. Currently, more than 350 genome sequences of SARS-CoV-2 available from the global initiative on sharing all influenza data (GISAID). The SARS-CoV-2 virus is a human infecting beta coronavirus, divergent from SARS-CoV, and that cause epidemic in 2003 (Gorbalenya et al. 2020).

Based on structural and biochemical studies, SARS-CoV-2, bind to human receptor Angiotensin-Converting enzyme-2 (ACE-2), through densely glycosylated spike protein (S) of SARS-CoV-2, is the initiation step to enter human cells. The binding of the spike protein of 
SARS-CoV-2 with human receptor ACE-2, through the receptor-binding domain (RBD) present in spike protein, is the most variable part of the coronavirus genome and form an RBD-ACE-2 complex (Andersen, Rambaut, Lipkin, Holmes \& Garry 2020). The amino acid sequence of RBD of SARS CoV-2 is different from SARS-CoV, and critical binds with more high affinity with human ACE-2 (Chen, Hotez \& Bottazzi 2020). $2.45 \AA$ resolution crystal structure of SARS$\mathrm{CoV}-2 \mathrm{~S}$ protein has been published (PDB: 6M0J). Based on this structure and solvent accessible binding surface is used for the tedious target for virtual screening and drug discovery (Jakhmola Mani, Sehgal, Dogra, Saxena \& Pande Katare 2020; Lan et al. 2020). The number of available $\mathrm{X}$-ray structures of SARS-CoV-2 RBD of $\mathrm{S}$ protein binding with various inhibitors is continuously reported in the protein data bank.

Protease enzyme $\left(\mathrm{M}^{\mathrm{Pro}}\right.$, also called $\left.3 \mathrm{CL}^{\text {Pro }}\right)$ is one of the best enzymes for drug targeting among coronavirus, due to its essential role in polyprotein processing, which is translated from viral RNA. Recently, the X-ray crystal structure of SARS-CoV-2 $\mathrm{M}^{\text {Pro }}$ has been solved, provides an excellent platform for structure-based drug discovery (Douangamath et al. 2020; Jin et al. 2020). A considerable effort has been given to studying this protein to find a therapeutic drug against SARS-CoV-2. The purpose of this study is to use the X-ray structure of protease enzyme $\mathrm{M}^{\mathrm{Pro}}$ and RBD of S protein to carry out virtual screening with 16 selected compounds to identify beneficial therapeutic compounds.

\section{Computational Details}

Molecular Docking Analysis: To understand the binding affinity and the intermolecular interaction of selected drug molecules with the active site amino acids of the main protease enzyme $\mathrm{M}^{\mathrm{Pro}}$ and $\mathrm{RBD}$ of $\mathrm{S}$ protein, molecular docking has been performed. Before docking, selected drug molecules were prepared by the Ligprep module of Schrodinger software. Further, 
these molecules were docked with the main protease enzyme $\mathrm{M}^{\text {Pro }}$ ( PDB: 5RFW) and RBD of $\mathrm{S}$ protein (PDB: 6M0J) by extra precision mode (XP) incorporated in the induced fit docking (IFD) method (Mirza, Salmas, Fatmi \& Durdagi 2016). The IFD resulted in different conformations for each ligand-main protease enzyme $\mathrm{M}^{\text {Pro }}$ and RBD of S protein complexes. Based on the top score with the lowest energy conformation and intermolecular interactions, the ligand-protein complexes were selected for each case and used for further study. PyMOL software was used to analyze the intermolecular interactions between ligand and protein for the individual complex.

Molecular dynamics simulation: To understand the stability, intermolecular interactions, and the binding energy of the selected two complexes of main protease enzyme $\mathrm{M}^{\text {Pro }}$ and RBD of $\mathrm{S}$ protein, the molecular dynamics (MD) simulation have been performed using the OPLS3e force field implemented in Desmond v5 package. Further, the system was built with the pre-defined TIP4P water model and orthorhombic periodic boundary conditions at the distances $10 \AA$. Then, the counter-ions were used to neutralize the charges of these complexes with the balancing $\mathrm{Na}+/ \mathrm{Cl}-$ ions. Further, the constructed system for each ligand with main protease enzyme $\mathrm{M}^{\text {Pro }}$ and RBD of S protein complexes were energy minimized by heating and equilibrium processes before the MD simulation. In the minimization step, the minimization and heating protocols were fixed based on the steepest descent method, annealing temperature at 0-300 K, and 2000 steps with the time steps of 0.001 ps. Further, the system was normalized in an equilibrium state at 1000 steps with a time step of 0.001 ps. Finally, the production step of the systems was continued up to $100 \mathrm{~ns}$ with the time steps of $0.001 \mathrm{ps,} 300 \mathrm{~K}, 1 \mathrm{~atm}$ pressure, and applied using the Nose-Hoover method with NPT ensemble (Prachanronarong et al. 2016; Rajendran, Shukla, Shukla \& Tripathi 2018). Intermolecular interactions and conformation of each ligand-protein complexes were analyzed from the final results of MD simulation (Elmezayen, Al-Obaidi, Şahin 
\& Yelekçi 2020; Mittal, Kumari, Srivastava, Singh \& Asthana 2020). Among the 1000 fractions, $5,10,15,20,25,30,35,40,45$, and 50 ns fractions were used to determine the binding free energy (MMGBSA) of the protein-ligand complexes by Prime application available in Schrodinger software package.

\section{Results and Discussion}

Binding mode and Intermolecular Interactions of $\mathbf{M}^{\text {pro }}$ protein with Indinavir: Several days has been predicted for treatment of COVID-19 recently including antivirals such as lopinavir, ritonavir, ribavirin, telbivudine, remidesvir, etc (Loustaud-Ratti et al. 2016; Cao et al. 2020; Cao, Deng \& Dai 2020; Dong, Hu \& Gao 2020; Kandeel \& Al-Nazawi 2020; Liu et al. 2020; Muralidharan, Sakthivel, Velmurugan \& Gromiha 2020; Wang et al. 2020). The available PDB structures of $\mathrm{M}^{\text {pro }}$ show the hydrogen bond interaction between the ligand T8M 405 and aminoacid residues $\mathrm{C} 145, \mathrm{G} 143$, and $\mathrm{H} 41$ of the protein. $\mathrm{H} 41$ also showed pi-cation interaction with the benzyl ring of the ligand (Douangamath et al. 2020; Jin et al. 2020). Ribavirin formed two hydrogen bonds with Thr25 and Gln 189 whereas, the residues Gln189 and Ser46 interact with the antiviral drug telbiduvine. Based on these data, the amino acid residues Cys145, Gly143, His41, Gln189, Ser46, and Thr25 are predicted as the key residues for the binding of antiviral compounds (Kandeel \& Al-Nazawi 2020).

15 antiviral compounds and the natural compound quercetin were docked using the induced fit docking method in the Schrodinger maestro suite. The drug indinavir showed a high binding affinity of -683.7 forming 3 hydrogen bonds between the ligand and His41, Ser46, and Asn142 residues of the $\mathrm{M}^{\mathrm{pro}}$ protein. Similarly, oseltamivir formed hydrogen bonds with Gln189, His 164, Asn142, and Gly143. Famciclavir formed 6 hydrogen bonds with Asn142, Gly143, 
His41, Thr25, and Glu166. The IFD score of oseltamivir and famciclavir were -681.909 and 683.411 respectively. The natural phytochemical quercetin formed 5 hydrogen bonds. Glu166 formed with the hydroxyl group of the benzyl ring, Gly143 interacts with keto and hydroxyl group of the aromatic ring. Cys145 with the keto group, Thr26 with the hydroxyl group. Also, His41 forms pi-cation interaction. Similarly, all the compounds with the hydrogen bond-forming residues were mentioned in Table 1 . The antiviral drug Tromantadine didn't show any significant binding with the $\mathrm{M}^{\text {pro }}$ enzyme. Figure 2 shows the superimposed form of all the drug molecules in the active site of Main Protease which reveals the orientation of the molecules and the

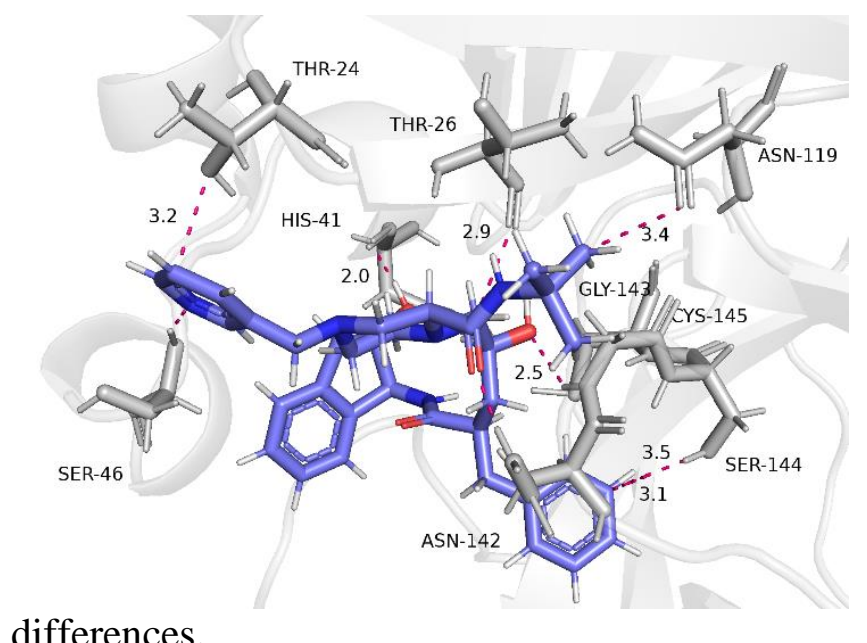

differences.

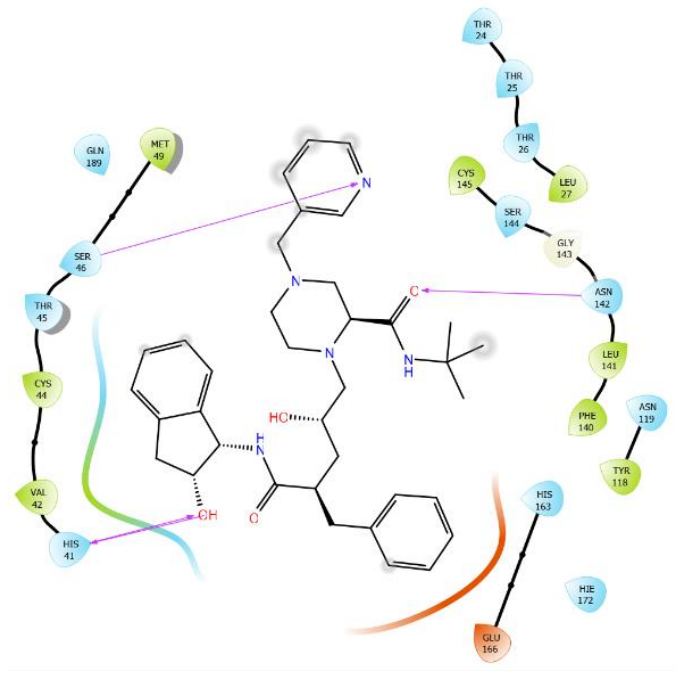

(b)

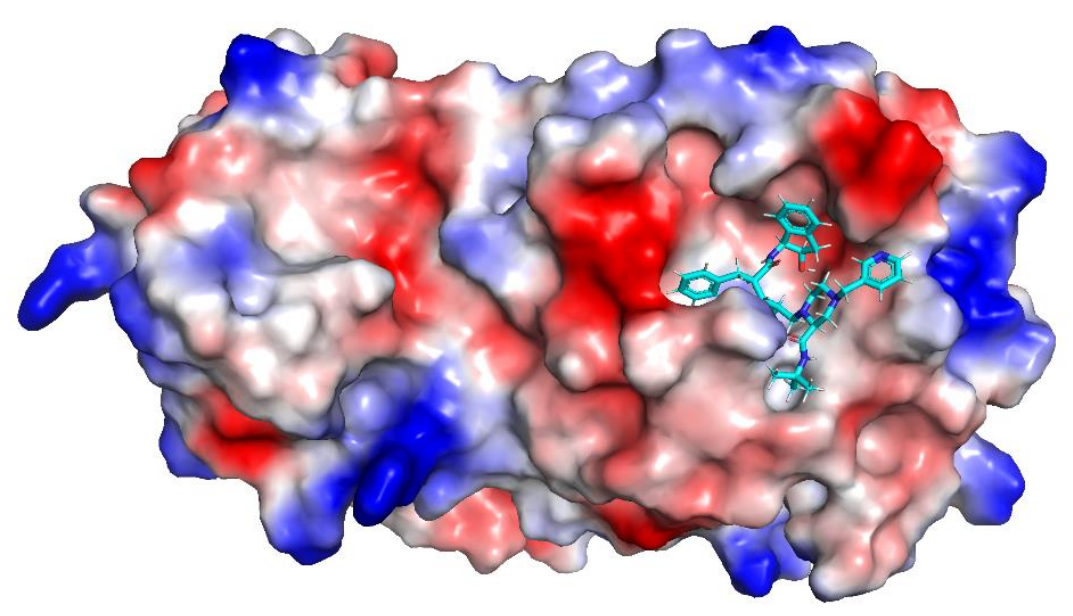


(c)

Figure 1: (a) Intermolecular interactions between Indinavir and active site residues of $\mathbf{M}^{\text {Pro }}$, (b) $2 \mathrm{D}$

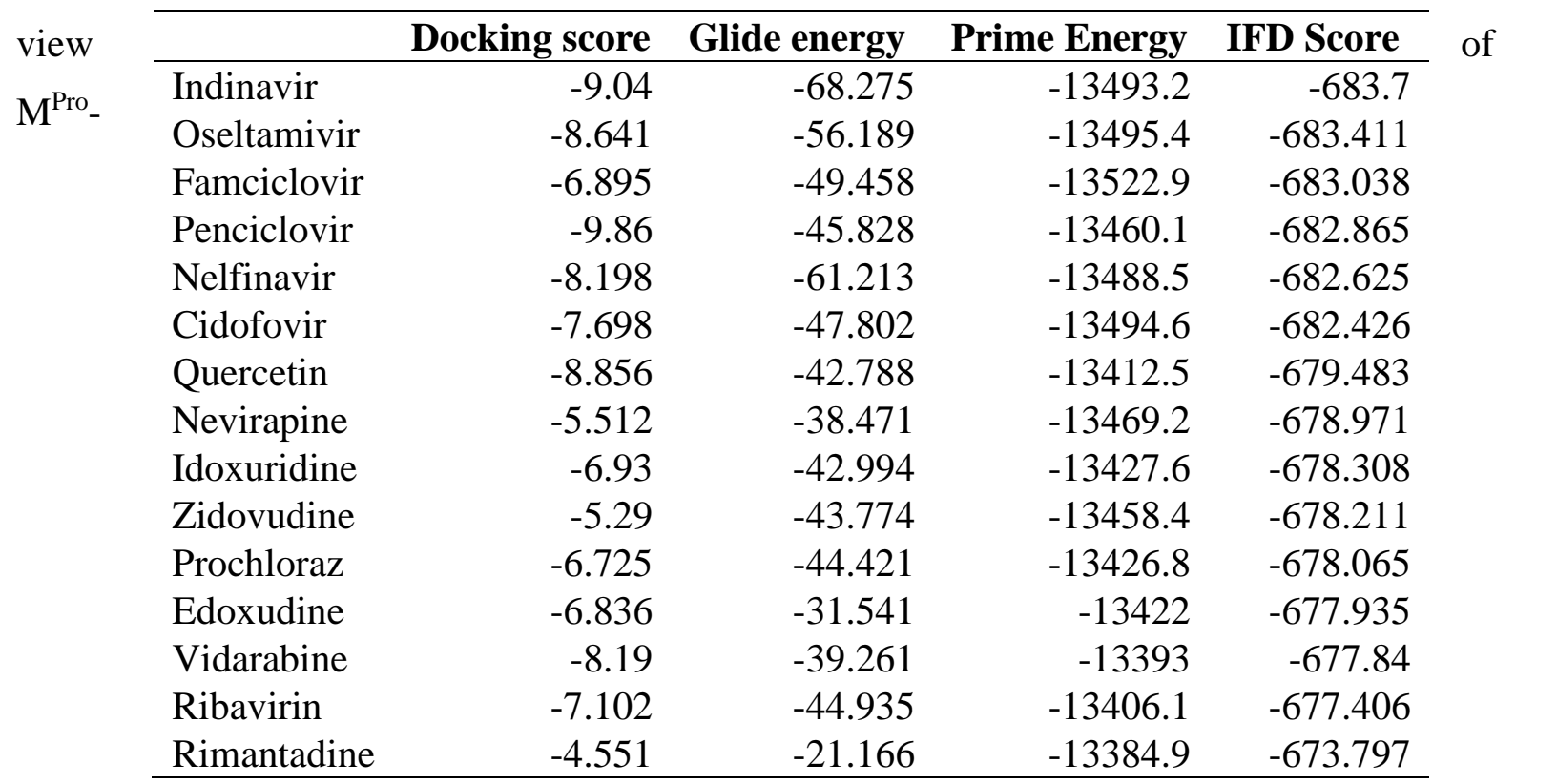

Indinavir complex, and (c) Electrostatic potential map of $\mathrm{M}^{\text {Pro }}$ with Indinavir molecule present in the active site cavity. 
Table 1: Different score values obtained from molecular docking of selected viral compounds with the main protease of SARS-2 CoV.

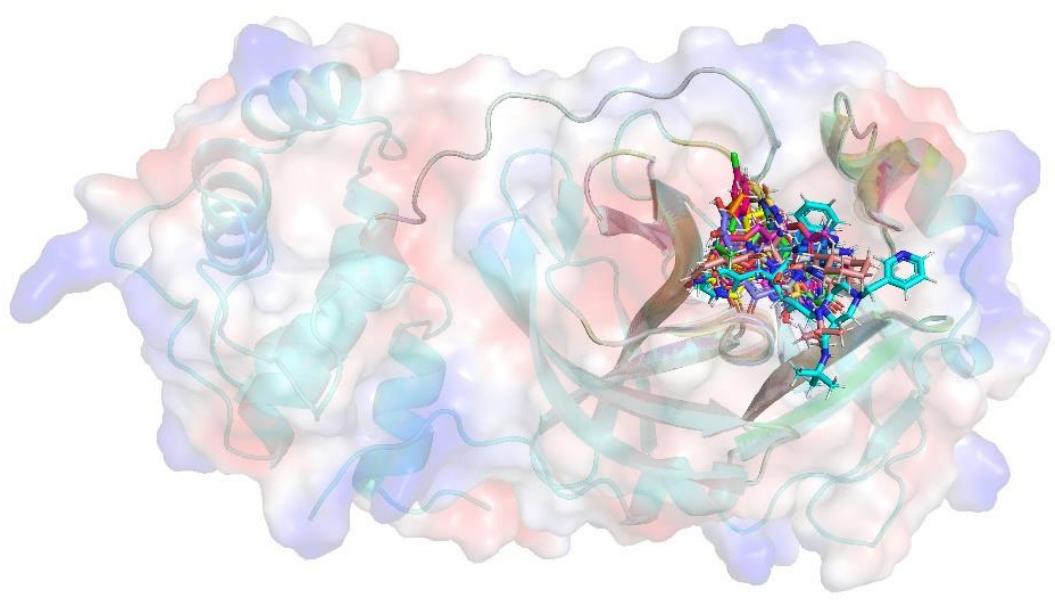

Figure 2: The superimposed form of all the selected drug molecules in the active site of Main Protease which reveals the orientation of the molecules and the differences.

Binding mode and Intermolecular Interactions of RBD of S protein with famciclavir: The S-protein of SARS-CoV-2 is most responsible for the virus entry through the binding of the receptor-binding domain of S-protein with ACE2 enzyme (Yan et al. 2020). Reportedly, by inhibiting the RBD of S-protein, the SARS-CoV-2 fusion can be stopped. In RBD of S-protein, the protein intermediate residues are playing a major role in viral entry. Notably, the surface residues like Gln493, Gly496, Gln498, and Asn502 are forming strong interactions with the ACE2 enzyme. Hence, to inhibit the function of this enzyme, the focused inhibitors should be interacting with these key residues (Hoffmann et al. 2020). The molecular docking of selected antiviral drugs with RBD of S-protein has been performed using the induced-fit docking (IFD) method. From the docking results, the docking score and IFD score values of famciclovir show a high binding affinity towards RBD of S-protein, the values are $-7.79 \mathrm{kcal} / \mathrm{mol}$ and -430.20 $\mathrm{kcal} / \mathrm{mol}$ respectively. The difference of docking scores and glide energy confirms the nature of 
their intermolecular interactions with the neighboring amino acids present in the active site of RBD of S-protein. All the selected anti-viral drugs are forming strong interactions with intermediate surface residues; particularly, the Gly496 is forming strong hydrogen bonding interaction with all compounds except indinavir and nevirapine which confirms all the selected compounds are binding with the target region. Moreover, the residues Tyr453, Gln498, Asn501 are also shown hydrogen bonding with selected compounds. In the famciclovir-RBD of S-protein complex, Tyr453, Gln493, Ser494, Gly496, and Tyr505 are showing strong interactions, the distances are 2.0, 2.1, 2.0, 2.1, and 3.3 A respectively. Based on the docking score, IFD score and intermolecular interactions, the famciclovir- RBD of the S-protein complex has been selected for further study. Figure 4 shows the superimposed form of all the drug molecules in the active site of RBD of S-protein which reveals the orientation of the molecules and the differences. Further, we have probed the existence of these interactions and binding stability of the famciclovir drug molecules with the RBD of S-protein during the MD simulations.

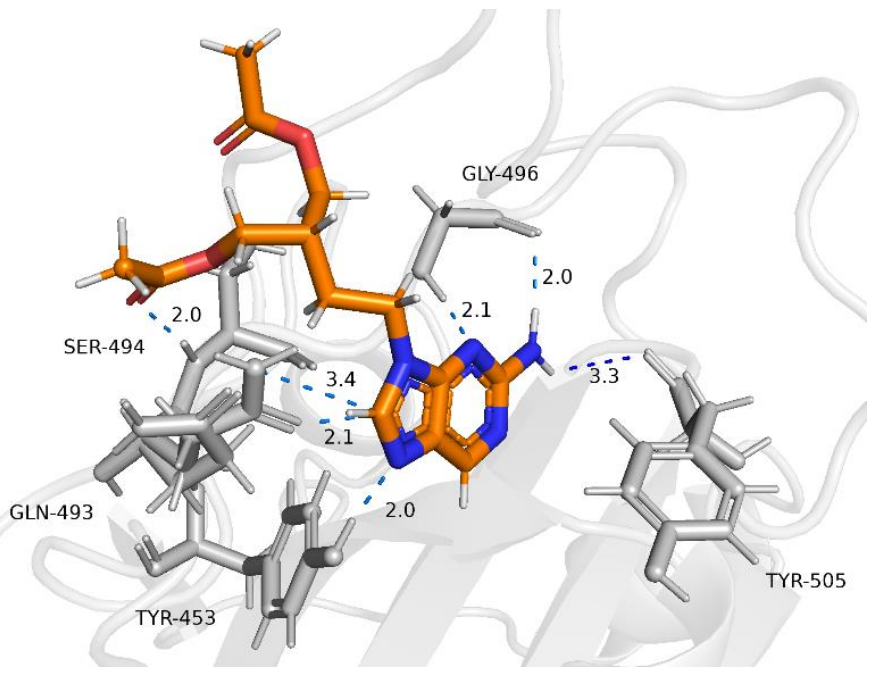

(a)
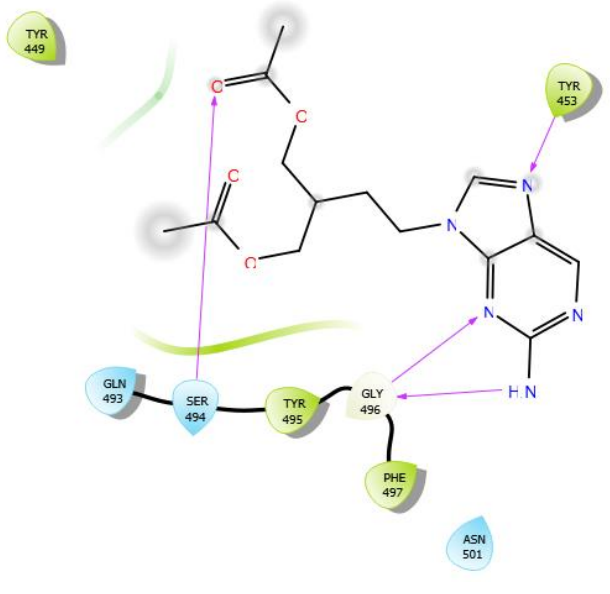

(b) 


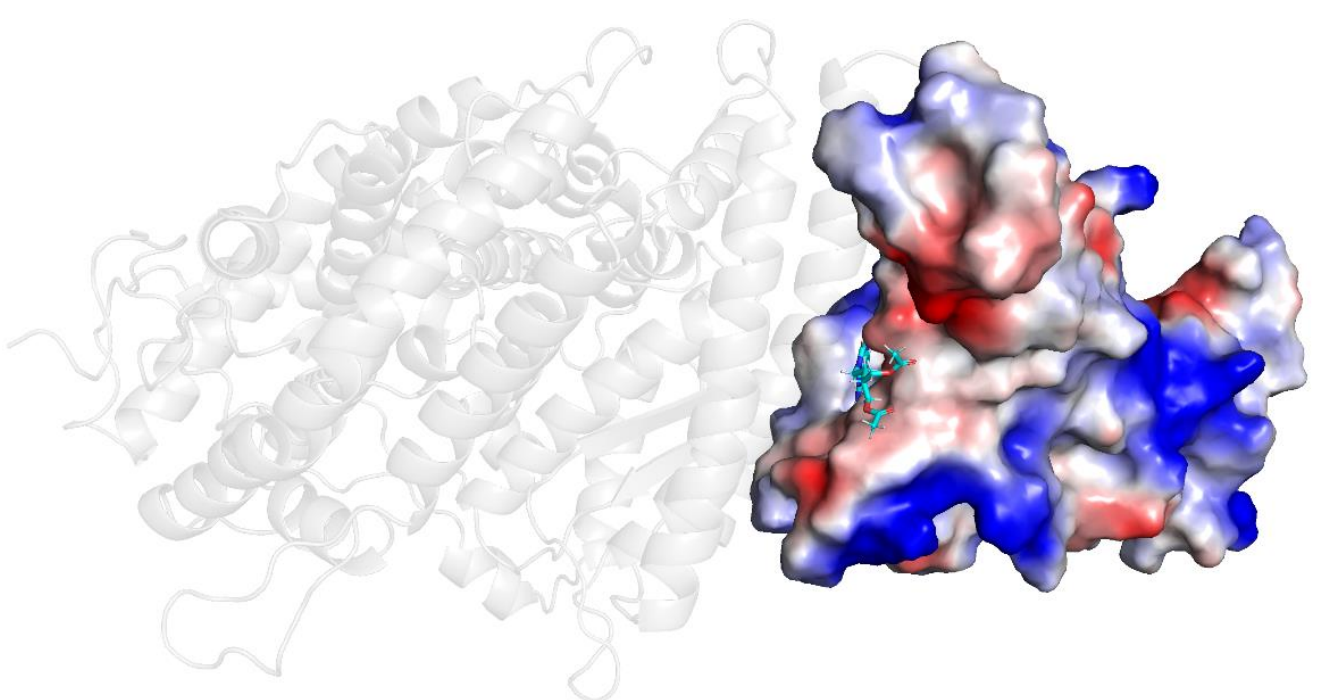

(c)

Figure 3: (a) Intermolecular interactions between famciclavir and interface residues of RBD of Spike protein, (b) 2D planer view of RBD- famciclavir complex, and (c) Electrostatic potential map of RBD- famciclavir with ACE2 (gray) protein. 


\begin{tabular}{lrrrr}
\hline & Docking score & Glide energy & Prime energy & IFD score \\
\hline Famciclovir & -7.793 & -40.518 & -8448.2 & -430.203 \\
Cidofovir & -7.943 & -35.938 & -8412.8 & -428.586 \\
Indinavir & -7.14 & -56.33 & -8383.3 & -426.306 \\
Edoxudine & -7.983 & -35.759 & -8363.8 & -426.175 \\
Penciclovir & -6.602 & -40.879 & -8362 & -424.703 \\
Oseltamivir & -4.155 & -32.188 & -8398.4 & -424.076 \\
Idoxuridine & -6.122 & -32.999 & -8354.7 & -423.857 \\
Nevirapine & -4.531 & -27.816 & -8385.5 & -423.806 \\
Nelfinavir & -4.943 & -42.28 & -8367.5 & -423.317 \\
Quercetin & -6.533 & -32.259 & -8332.4 & -423.155 \\
Zidovudine & -4.493 & -32.356 & -8370.2 & -423.004 \\
Ribavirin & -7.578 & -36.097 & -8303.3 & -422.743 \\
Prochloraz & -4.793 & -32.146 & -8346.7 & -422.129 \\
Vidarabine & -5.606 & -32.579 & -8314 & -421.305 \\
Rimantadine & -3.39 & -18.111 & -8323.6 & -419.57 \\
Tromantadine & -3.34 & -27.093 & -8312.2 & -418.951 \\
\hline
\end{tabular}

Table 2: Different score values obtained from molecular docking of selected viral compounds with receptor binding domain of Spike protein.

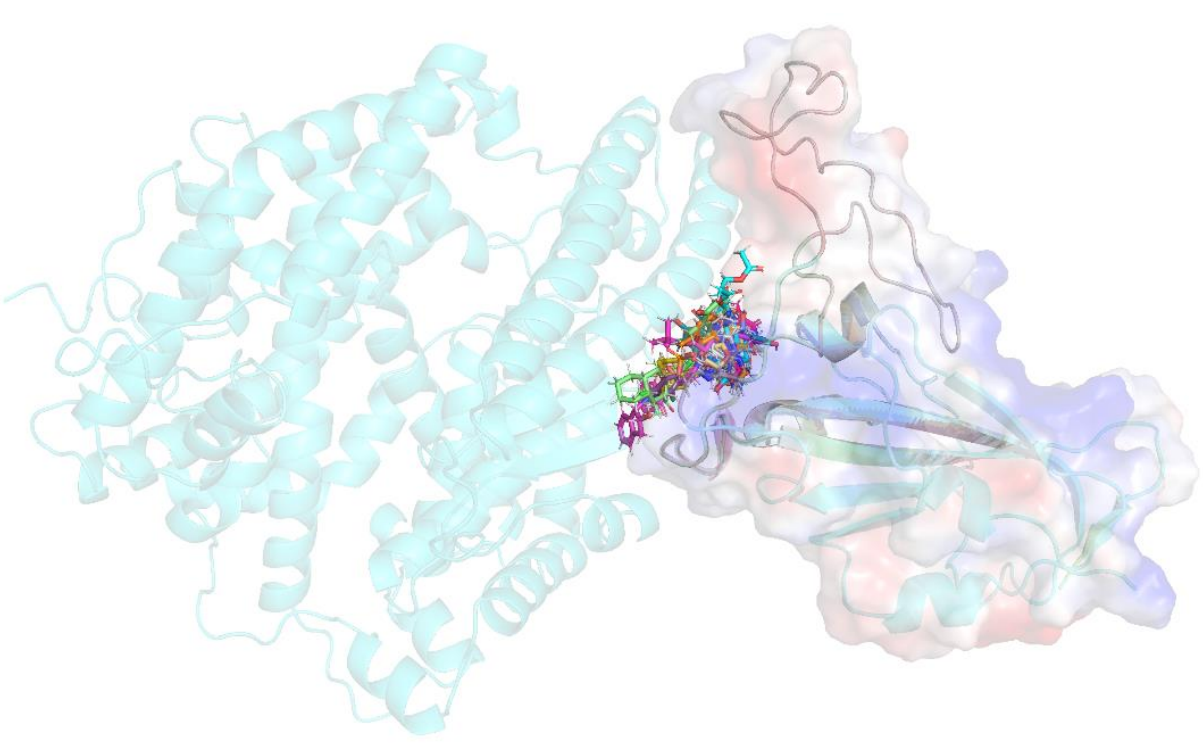

Figure 4: The superimposed form of all the drug molecules in the active site of RBD of S-protein which reveals the orientation of the molecules and the differences. 


\section{RMSD, RMSF, and Intermolecular Interactions from MD Simulation:}

Molecular dynamics help to understand the biological function of proteins and proteinligand complexes (Rajendran et al. 2018). Here we have performed 50 ns MD simulations for the and Indinavir- $\mathrm{M}^{\text {pro }}$ enzyme and famciclovir - RBD of S-protein complexes which is also helping to explore the stability and the binding affinity of the drug molecules in the corresponding protein environments. Figure 5 displays the statistical parameters (RMSD and RMSF) of the two complexes during the MD simulations. The RMSD values of the Indinavir- $\mathrm{M}^{\text {pro }}$ complex remains within $2.5 \AA$ (Figure 5a). Whereas the RMSD of famciclovir - RBD of S-protein complex shows $2.7 \AA$ and is found to be high on compare with Indinavir- $\mathrm{M}^{\text {pro }}$ complex. Notably, the complex famciclovir - RBD of S-protein was stabilized after 30 ns of MD simulation (Fig 5c). Figure 5b\&d shows the variations of RMSF of amino acids of two proteins; among these, high fluctuations are found in the loop region than the $\alpha$-helix and $\beta$ sheet. The superimposed form of the three ligand-protein complexes (Figure 6) obtained from the docking and MD simulations are showing the conformational difference of both ligands and proteins in the respective complexes; specifically, this allows to visualize that how the conformation and the orientation of the ligands and proteins are altered during the MD simulation. Overall, the fluctuation of the indinavir-Mpro complex is found to be very less and it is normal.
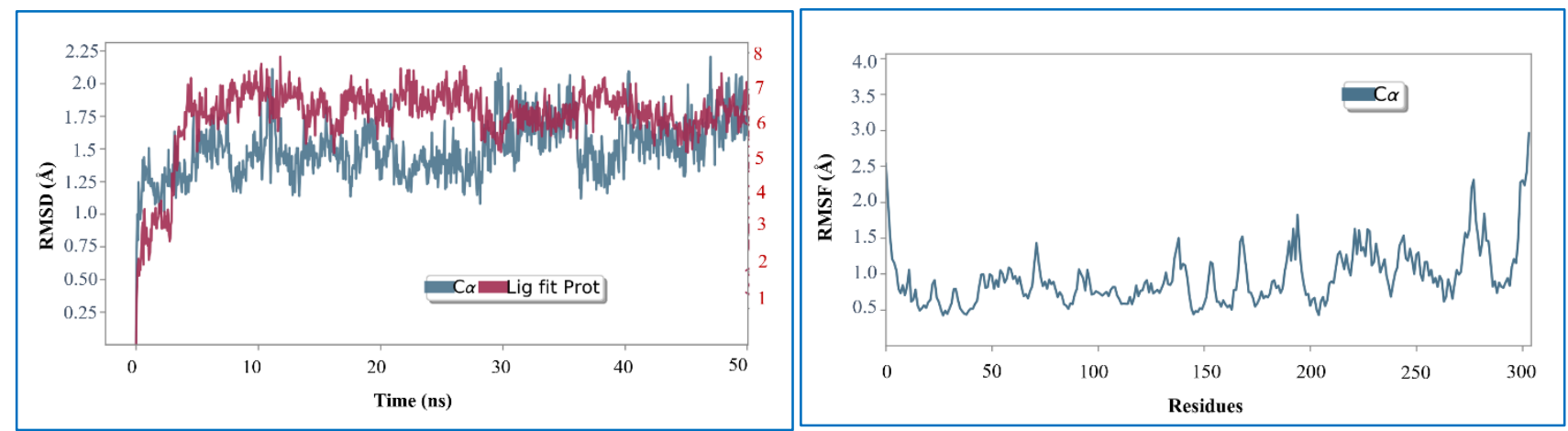
(a)

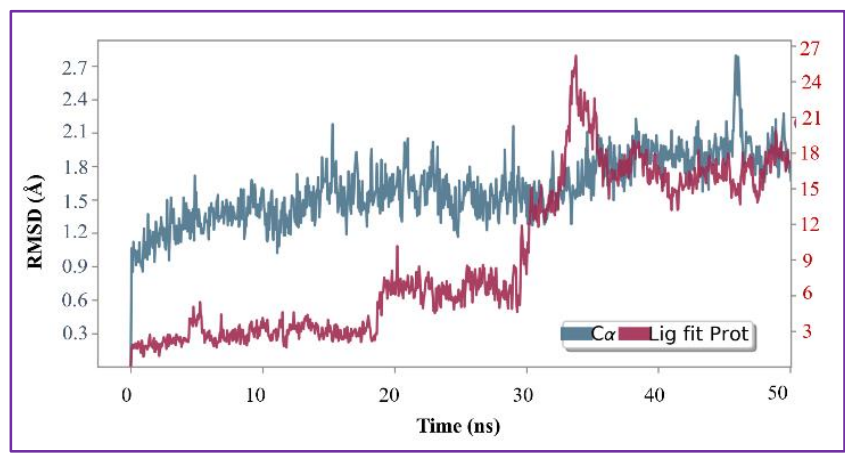

(c) (b)

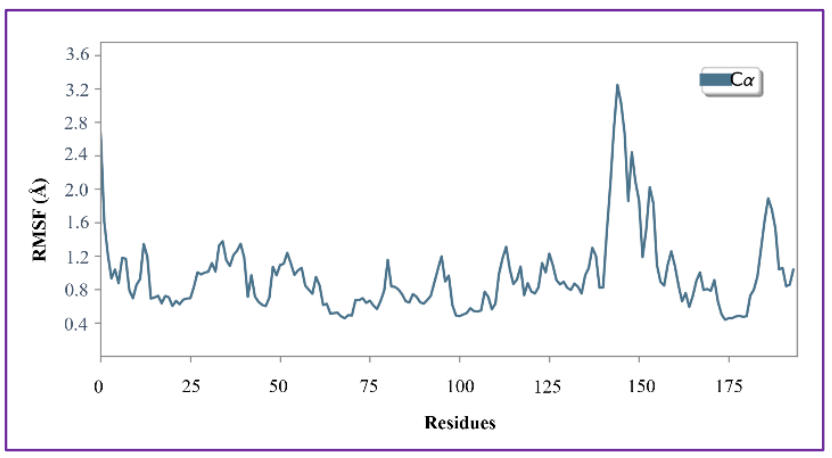

(d)

Figure 5: RMSD and RMSF of (a,b) Indinavir-MPro complex and (c,d) famciclovir - RBD of Sprotein complex respectively.
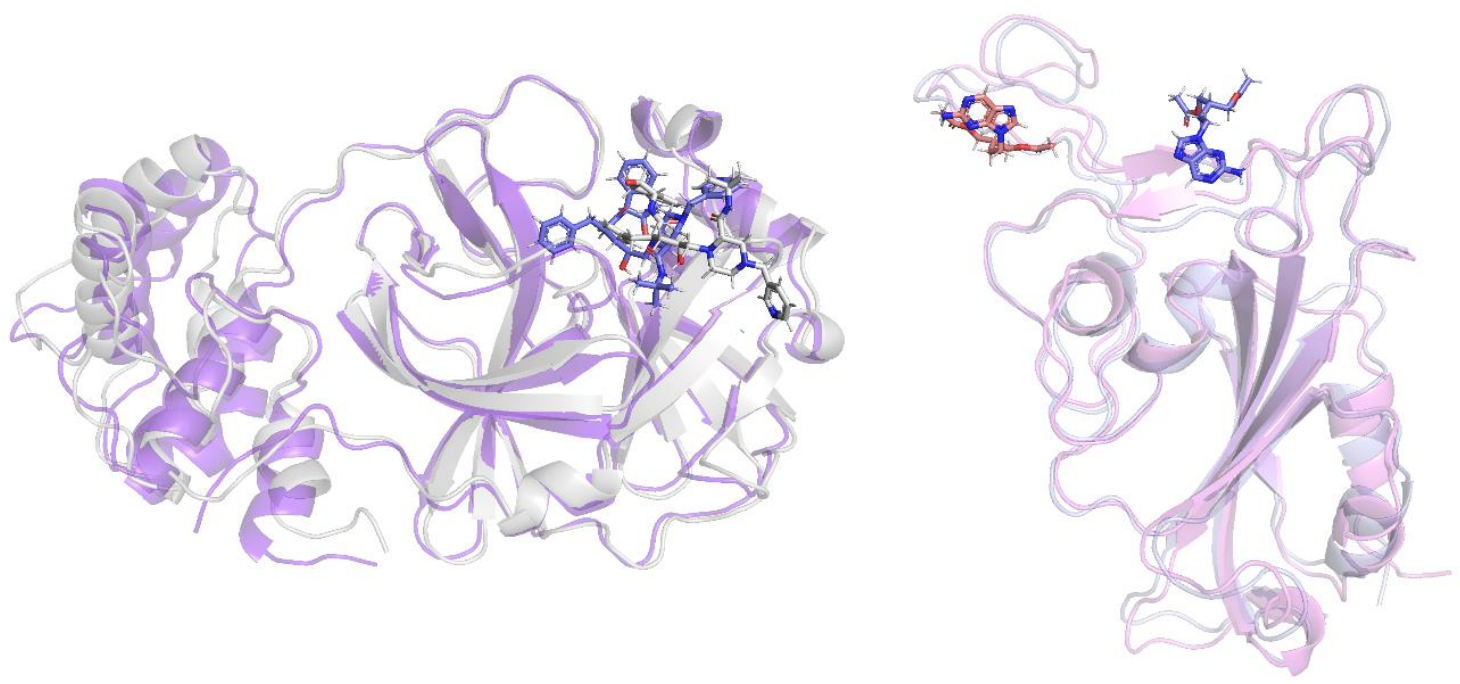

Figure 6: Superimposed of Indinavir-MPro complex and (c,d) famciclovir - RBD of S-protein complexes from docking and MD simulation.

Intermolecular interactions were analyzed after $50 \mathrm{~ns}$ of MD simulation; in which, intermolecular interactions such as hydrogen bonds, hydrophobic, and water bridge interactions exist in the indinavir-MPro complex after $50 \mathrm{~ns}$ of MD simulations. Notably, the residues Thr24 and Thr26 is forming strong hydrogen-bonding interactions with indinavir molecule during the simulation which are highly stable (92 and $86 \%$ ) throughout the simulation. Moreover, the amino acid Asn119 is forming water bridge interaction with an indinavir molecule. Whereas in 
famciclovir - RBD of S-protein complex, the residue Tyr453 is maintained their interaction with famciclovir molecule up to $50 \mathrm{~ns}$ of MD simulation. Furthermore, MMGBSA was calculated for these two complexes that are $-74.949 \mathrm{kcal} / \mathrm{mol}$ and $-31.776 \mathrm{kcal} / \mathrm{mol}$ respectively. Therefore from these results, we concluded that these two molecules with $\mathrm{M}^{\mathrm{Pro}}$ and RBD of S-protein were highly stable during the simulation and it could be potential inhibitors and we can also design new compounds from these known drugs.

\section{Conclusion}

The major source of disaster in the 21 st century is the 2019-novel coronavirus disease (Covid19). In the past few months, the world is eagerly waiting for a solution and put a strong effort to find drug compounds against different targets responsible for Covid19. At this current point in time, the lack of specific drugs to either treat or prevent this world disaster. Therefore, we have selected 16 antiviral drugs and performed molecular docking and MD simulation to put a finger on against $\mathrm{M}^{\mathrm{Pro}}$ and $\mathrm{RBD}$ of the spike protein. From our results, the drug indinavir showed high binding affinity and strong hydrogen bonding with $\mathrm{M}^{\text {pro }}$ protein whereas, in the RBD of S-protein, the famciclovir shows high binding affinity and strong interactions. On comparing these results, we propose these two (indinavir and Famciclovir) drugs could be helpful against COVID-19 until new drugs are discovered.

\section{Acknowledgment}

We acknowledge Mr. Vinoth from Schrodinger, India for providing the Desmond results.

\section{References}

Andersen, K. G., Rambaut, A., Lipkin, W. I., Holmes, E. C. \& Garry, R. F. (2020). The proximal origin of SARS-CoV-2. Nature Medicine 26: 450-452. 
Cao, B., Wang, Y., Wen, D., Liu, W., Wang, J., Fan, G., Ruan, L., Song, B., Cai, Y., Wei, M., Li, X., Xia, J., Chen, N., Xiang, J., Yu, T., Bai, T., Xie, X., Zhang, L., Li, C., Yuan, Y., Chen, H., Li, H., Huang, H., Tu, S., Gong, F., Liu, Y., Wei, Y., Dong, C., Zhou, F., Gu, X., Xu, J., Liu, Z., Zhang, Y., Li, H., Shang, L., Wang, K., Li, K., Zhou, X., Dong, X., Qu, Z., Lu, S., Hu, X., Ruan, S., Luo, S., Wu, J., Peng, L., Cheng, F., Pan, L., Zou, J., Jia, C., Wang, J., Liu, X., Wang, S., Wu, X., Ge, Q., He, J., Zhan, H., Qiu, F., Guo, L., Huang, C., Jaki, T., Hayden, F. G., Horby, P. W., Zhang, D. \& Wang, C. (2020). A Trial of Lopinavir-Ritonavir in Adults Hospitalized with Severe Covid-19. 382: 1787-1799.

Cao, Y.-c., Deng, Q.-X. \& Dai, S.-X. (2020). Remdesivir for severe acute respiratory syndrome coronavirus 2 causing COVID-19: An evaluation of the evidence. Travel Medicine and Infectious Disease 35: 101647.

Chen, W.-H., Hotez, P. J. \& Bottazzi, M. E. (2020). Potential for developing a SARS-CoV receptor-binding domain $(\mathrm{RBD})$ recombinant protein as a heterologous human vaccine against coronavirus infectious disease (COVID)-19. Human Vaccines \& Immunotherapeutics 16: 1239-1242.

Cui, J., Li, F. \& Shi, Z.-L. (2019). Origin and evolution of pathogenic coronaviruses. Nature Reviews Microbiology 17: 181-192.

Dong, L., Hu, S. \& Gao, J. (2020). Discovering drugs to treat coronavirus disease 2019 (COVID19). Drug Discoveries \& Therapeutics 14: 58-60.

Douangamath, A., Fearon, D., Gehrtz, P., Krojer, T., Lukacik, P., Owen, C. D., Resnick, E., Strain-Damerell, C., Aimon, A., Ábrányi-Balogh, P., Brandaõ-Neto, J., Carbery, A., Davison, G., Dias, A., Downes, T. D., Dunnett, L., Fairhead, M., Firth, J. D., Jones, S. P., Keely, A., Keserü, G. M., Klein, H. F., Martin, M. P., Noble, M. E. M., O’Brien, P., Powell, A., Reddi, R., Skyner, R., Snee, M., Waring, M. J., Wild, C., London, N., von Delft, F. \& Walsh, M. A. (2020). Crystallographic and electrophilic fragment screening of the SARS-CoV-2 main protease. 2020.2005.2027.118117.

Elmezayen, A. D., Al-Obaidi, A., Şahin, A. T. \& Yelekçi, K. (2020). Drug repurposing for coronavirus (COVID-19): in silico screening of known drugs against coronavirus 3CL hydrolase and protease enzymes. Journal of Biomolecular Structure and Dynamics: 1-13. 
Gorbalenya, A. E., Baker, S. C., Baric, R. S., de Groot, R. J., Drosten, C., Gulyaeva, A. A., Haagmans, B. L., Lauber, C., Leontovich, A. M., Neuman, B. W., Penzar, D., Perlman, S., Poon, L. L. M., Samborskiy, D. V., Sidorov, I. A., Sola, I., Ziebuhr, J. \& Coronaviridae Study Group of the International Committee on Taxonomy of, V. (2020). The species Severe acute respiratory syndrome-related coronavirus: classifying 2019$\mathrm{nCoV}$ and naming it SARS-CoV-2. Nature Microbiology 5: 536-544.

Hoffmann, M., Kleine-Weber, H., Schroeder, S., Krüger, N., Herrler, T., Erichsen, S., Schiergens, T. S., Herrler, G., Wu, N.-H., Nitsche, A., Müller, M. A., Drosten, C. \& Pöhlmann, S. (2020). SARS-CoV-2 Cell Entry Depends on ACE2 and TMPRSS2 and Is Blocked by a Clinically Proven Protease Inhibitor. Cell 181: 271-280.e278.

Jakhmola Mani, R., Sehgal, N., Dogra, N., Saxena, S. \& Pande Katare, D. (2020). Deciphering underlying mechanism of Sars-CoV-2 infection in humans and revealing the therapeutic potential of bioactive constituents from Nigella sativa to combat COVID19: in-silico study. Journal of Biomolecular Structure and Dynamics: 1-13.

Jin, Z., Du, X., Xu, Y., Deng, Y., Liu, M., Zhao, Y., Zhang, B., Li, X., Zhang, L., Peng, C., Duan, Y., Yu, J., Wang, L., Yang, K., Liu, F., Jiang, R., Yang, X., You, T., Liu, X., Yang, X., Bai, F., Liu, H., Liu, X., Guddat, L. W., Xu, W., Xiao, G., Qin, C., Shi, Z., Jiang, H., Rao, Z. \& Yang, H. (2020). Structure of Mpro from SARS-CoV-2 and discovery of its inhibitors. Nature 582: 289-293.

Kandeel, M. \& Al-Nazawi, M. (2020). Virtual screening and repurposing of FDA approved drugs against COVID-19 main protease. Life Sciences 251: 117627.

Lan, J., Ge, J., Yu, J., Shan, S., Zhou, H., Fan, S., Zhang, Q., Shi, X., Wang, Q., Zhang, L. \& Wang, X. (2020). Structure of the SARS-CoV-2 spike receptor-binding domain bound to the ACE2 receptor. Nature 581: 215-220.

Lathika Rajendrakumar, A., Thakarakkattil Narayanan Nair, A., Nangia, C., Kumar Chourasia, P., Kumar Chourasia, M., Ghouse Syed, M., Sasidharan Nair, A., B Nair, A. \& Fazaludeen Koya, M. S. (2020). Epidemic Landscape and Forecasting of SARS-CoV-2 in India. medRxiv: 2020.2004.2014.20065151. 
Liu, F., Xu, A., Zhang, Y., Xuan, W., Yan, T., Pan, K., Yu, W. \& Zhang, J. (2020). Patients of COVID-19 may benefit from sustained Lopinavir-combined regimen and the increase of Eosinophil may predict the outcome of COVID-19 progression. International Journal of Infectious Diseases 95: 183-191.

Loustaud-Ratti, V., Debette-Gratien, M., Jacques, J., Alain, S., Marquet, P., Sautereau, D., Rousseau, A. \& Carrier, P. (2016). Ribavirin: Past, present and future. World journal of hepatology 8: 123-130.

Mirza, S. B., Salmas, R. E., Fatmi, M. Q. \& Durdagi, S. (2016). Virtual screening of eighteen million compounds against dengue virus: Combined molecular docking and molecular dynamics simulations study. Journal of Molecular Graphics and Modelling 66: 99-107.

Mittal, L., Kumari, A., Srivastava, M., Singh, M. \& Asthana, S. (2020). Identification of potential molecules against COVID-19 main protease through structure-guided virtual screening approach. Journal of Biomolecular Structure and Dynamics: 1-19.

Muralidharan, N., Sakthivel, R., Velmurugan, D. \& Gromiha, M. M. (2020). Computational studies of drug repurposing and synergism of lopinavir, oseltamivir and ritonavir binding with SARS-CoV-2 protease against COVID-19. Journal of Biomolecular Structure and Dynamics: 1-6.

Prachanronarong, K. L., Özen, A., Thayer, K. M., Yilmaz, L. S., Zeldovich, K. B., Bolon, D. N., Kowalik, T. F., Jensen, J. D., Finberg, R. W., Wang, J. P., Kurt-Yilmaz, N. \& Schiffer, C. A. (2016). Molecular Basis for Differential Patterns of Drug Resistance in Influenza N1 and N2 Neuraminidase. Journal of Chemical Theory and Computation 12: 6098-6108.

Rajendran, V., Shukla, R., Shukla, H. \& Tripathi, T. (2018). Structure-function studies of the asparaginyl-tRNA synthetase from Fasciola gigantica: understanding the role of catalytic and non-catalytic domains. Biochemical Journal 475: 3377-3391.

Wang, Y., Zhang, D., Du, G., Du, R., Zhao, J., Jin, Y., Fu, S., Gao, L., Cheng, Z., Lu, Q., Hu, Y., Luo, G., Wang, K., Lu, Y., Li, H., Wang, S., Ruan, S., Yang, C., Mei, C., Wang, Y., Ding, D., Wu, F., Tang, X., Ye, X., Ye, Y., Liu, B., Yang, J., Yin, W., Wang, A., Fan, G., Zhou, F., Liu, Z., Gu, X., Xu, J., Shang, L., Zhang, Y., Cao, L., Guo, T., Wan, Y., Qin, 
H., Jiang, Y., Jaki, T., Hayden, F. G., Horby, P. W., Cao, B. \& Wang, C. (2020). Remdesivir in adults with severe COVID-19: a randomised, double-blind, placebocontrolled, multicentre trial. The Lancet 395: 1569-1578.

World Health Organization (2020). Coronavirus disease 2019 (COVID-19): situation report, 72, World Health Organisation.

Yan, R., Zhang, Y., Li, Y., Xia, L., Guo, Y. \& Zhou, Q. (2020). Structural basis for the recognition of SARS-CoV-2 by full-length human ACE2. Science 367: 1444-1448.

Yang, X., Yu, Y., Xu, J., Shu, H., Xia, J. a., Liu, H., Wu, Y., Zhang, L., Yu, Z., Fang, M., Yu, T., Wang, Y., Pan, S., Zou, X., Yuan, S. \& Shang, Y. (2020). Clinical course and outcomes of critically ill patients with SARS-CoV-2 pneumonia in Wuhan, China: a singlecentered, retrospective, observational study. The Lancet Respiratory Medicine 8: 475481 . 